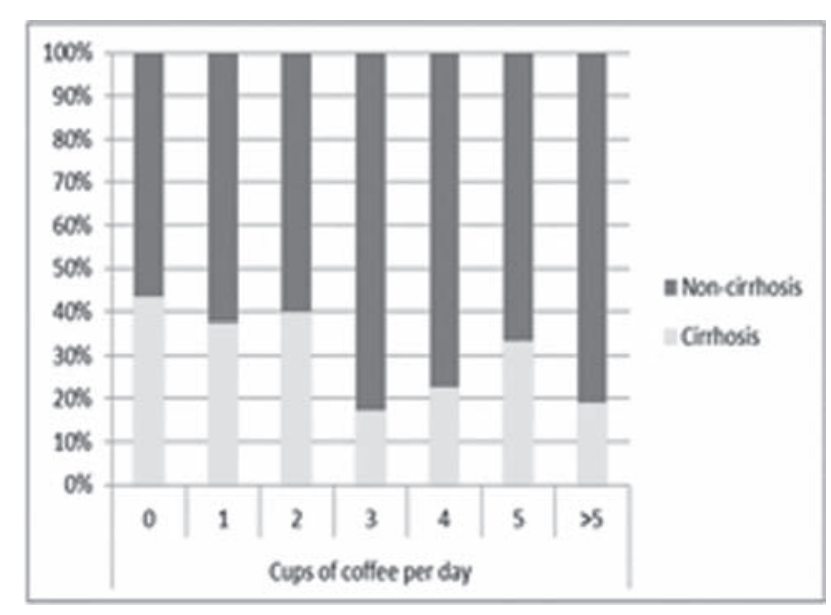

Figure 1 PTH-107 Cups of coffee per day

patients and controls was not significant. There was no correlation between Child Pugh and MELD scores and coffee consumption ( -0.12 and -0.02 , respectively).

Conclusion Coffee consumption appears to have a protective effect in the development of cirrhosis in liver disease. However, the results did not give a significant difference in the amount of coffee drunk by liver patients and the control groups. It is possible that by changing lifestyle factors, including the amount of coffee drunk, the development of cirrhosis could be postponed.

Competing interests None.

Keywords cirrhosis, coffee, liver.

\section{PTH-107 $\quad$ STUDY OF COFFEE CONSUMPTION AND LIVER DISEASE}

doi:10.1136/gut.2011.239301.508

H B Walton, 1,* G Masterton, ${ }^{2}$ P C Hayes ${ }^{2}{ }^{1}$ Medical School, University of Edinburgh, Edinburgh, UK; ${ }^{2}$ Hepatology, Royal Infirmary of Edinburgh, Edinburgh, UK

Introduction Liver disease is the fifth most common cause of death in the UK. Previous studies have reported that drinking coffee can protect against the development of abnormal liver function tests, hepatic fibrosis and cirrhosis.

The aims of this study were to compare the coffee consumption of patients with liver disease and that of control populations in order to determine if there is a correlation between coffee intake and the incidence of non-cancerous liver disease.

Methods 274 patients attending the liver outpatient department at the Royal Infirmary of Edinburgh between June and September 2010 completed a questionnaire detailing coffee consumption and lifestyle factors. Case notes were reviewed for clinical information. Questionnaires were also completed by 120 medical students and 100 orthopaedic outpatients to act as control populations.

Results Of the 274 liver disease patients, 95 had established cirrhosis. A significant difference $(p=<0.05)$ was found in coffee consumption of patients with cirrhosis (2.11 cups per day) and those who were non-cirrhotic (3.62 cups per day) see figure 1 . The difference in coffee consumption by liver disease 